\title{
The relaxed micromorphic continuum model
}

\author{
Ionel-Dumitrel Ghiba ${ }^{1, *}$, Patrizio Neff ${ }^{2, * *}$, and Angela Madeo ${ }^{3, * * *}$ \\ ${ }^{1}$ Lehrstuhl für Nichtlineare Analysis und Modellierung, Fakultät für Mathematik, Universität Duisburg-Essen, \\ Thea-Leymann Str. 9, 45127 Essen, Germany; Alexandru Ioan Cuza University of Iaşi, Department of Mathematics, Blvd. \\ Carol I, no. 11, 700506 Iaşi, Romania; and Octav Mayer Institute of Mathematics of the Romanian Academy, Iaşi Branch, \\ 700505 Iaşi. \\ ${ }^{2}$ Head of Lehrstuhl für Nichtlineare Analysis und Modellierung, Fakultät für Mathematik, Universität Duisburg-Essen, \\ Thea-Leymann Str. 9, 45127 Essen, Germany \\ ${ }^{3}$ Laboratoire de Génie Civil et Ingénierie Environnementale, Université de Lyon-INSA, Bâtiment Coulomb, 69621 \\ Villeurbanne Cedex, France; and International Center M\&MOCS "Mathematics and Mechanics of Complex Systems", \\ Palazzo Caetani, Cisterna di Latina, Italy
}

\begin{abstract}
We formulate a relaxed linear elastic micromorphic continuum model with symmetric Cauchy force-stresses and curvature contribution depending only on the micro-dislocation tensor. We prove that the new relaxed micromorphic model supports well-posedness results. Relations with other theories are also pointed out.
\end{abstract}

(c) 2014 Wiley-VCH Verlag GmbH \& Co. KGaA, Weinheim

\section{Introduction}

In this paper we consider the relaxed micromorphic model introduced in [9]. The basic kinematical fields of this extended continuum model are the displacement $u \in \mathbb{R}^{3}$ and the non-symmetric micro-distortion density tensor $P \in \mathbb{R}^{3 \times 3}$. Our new model reconciles Kröner's rejection of antisymmetric force stresses in dislocation theory with the asymmetric dislocation model of Eringen and Claus [3] and shows that the concept of asymmetric force stress is not strictly needed in order to describe rotations of the microstructure in non-polar materials (see also [6]). Hence in our relaxed theory a symmetric force-stress tensor arises despite the presence of microstructure and the curvature contribution depends solely on the micro-dislocation tensor Curl $P \in \mathbb{R}^{3 \times 3}$. However, the relaxed model is able to fully describe rotations of the microstructure and to predict non-polar size-effects. Therefore, it gets clear that the asymmetry of the force stress tensor in a continuum theory is not an immediate consequence of the presence of microstructure in the body, it is rather a constitutive assumption. Another interesting feature concerns the prescription of boundary values for the micro-distortion field: only tangential traces may be determined which are weaker than the usual strong anchoring boundary condition. In contrast with the models considered until now, our free energy of the relaxed model is not uniformly pointwise positive definite in the control of the classical constitutive variables. We mention that the well-posedness of the dynamic problem of the relaxed model is established in [4], while a well-posed problem class of autonomous evolutionary equations from elasticity theory modeling solids with microstructure are studied in [13]. The existence results for the static geometrically nonlinear formulation are established in [7]. Some very special engineering meta-materials like phononic crystals and lattice structures may however need the introduction of asymmetric force stress to fully describe their mechanical behavior. This fact was observed in [5], in which the presence of the Cosserat couple modulus has been proved to be necessary for the physically plausible description of the dynamical behavior of high-tech micro-structured materials which are known to exhibit frequency band-gaps in the dynamic regime. The relaxed micromorphic model with positive Cosserat modulus proposed in [5] is the only generalized continuum model which is able to predict frequency band-gaps contrarily to what is possible in the Mindlin-Eringen model or in so-called second-gradient models.

\subsection{Formulation of the relaxed micromorphic model}

We consider a micromorphic continuum which occupies a bounded domain $\Omega$ and is bounded by the piecewise smooth surface $\partial \Omega$. Let $T>0$ be a given time and let $\operatorname{Sym}(3)$ denote the set of symmetric tensors. In this note we keep the notation and we consider the same Sobolev spaces as in [9]. We consider the following system of partial differential equations

$$
u_{, t t}=\operatorname{Div}[\underbrace{\mathbb{C} \cdot \operatorname{sym}(\nabla u-P)}_{\text {the Cauchy stress } \sigma \text { (symmetric) }}]+f, \quad P_{, t t}=\underbrace{-\operatorname{Curl}[\mathbb{L}_{c} \cdot \underbrace{\operatorname{Curl} P}_{\text {the dislocation density tensor }}]}_{\text {describes the micro-moments }}+\mathbb{C} \cdot \operatorname{sym}(\nabla u-P)-\underbrace{\mathbb{H} . \operatorname{sym} P}_{\text {the microstress tensor } s \text { (symmetric) }}+M \quad
$$

\footnotetext{
* Corresponding author: e-mail dumitrel.ghiba@uni-due.de,dumitrel.ghiba@uaic.ro

** email: patrizio.neff@uni-due.de

*** email: angela.madeo@insa-lyon.fr
} 
in $\Omega \times[0, T]$, where $f$ is the body force, $M$ is the body moment tensor (second order, non-symmetric), $\mathbb{C}: \operatorname{Sym}(3) \rightarrow \operatorname{Sym}(3)$, $\mathbb{H}: \operatorname{Sym}(3) \rightarrow \operatorname{Sym}(3), \mathbb{L}_{c}: \mathbb{R}^{3 \times 3} \rightarrow \mathbb{R}^{3 \times 3}$ are material tensors. To our system of partial differential equations we adjoin the weaker than usual boundary conditions: $u(x, t)=0$, and the tangential condition $P_{i}(x, t) \times n(x)=0, i=1,2,3$, on $\partial \Omega \times[0, T]$, where $\times$ denotes the vector product, $n$ is the unit outward normal vector at the surface $\partial \Omega, P_{i}, i=1,2,3$ are the rows of $P$. The model is driven by nonzero initial conditions $u(x, 0)=u_{0}(x), \dot{u}(x, 0)=\dot{u}_{0}(x), P(x, 0)=P_{0}(x), \dot{P}(x, 0)=$ $\dot{P}_{0}(x)$, in $\Omega$, where $u_{0}, \dot{u}_{0}, P_{0}$ and $\dot{P}_{0}$ are prescribed functions.

Among the advantages of our relaxed model we point out that in contrast with the 7+11 parameters isotropic Mindlin and Eringen model, we have altogether only seven parameters. Another advantage is that we do not use the strong anchoring condition, $P(x, t)=0$ on $\partial \Omega$. Moreover, by suitable restriction of the model we obtain (see [9]): the linear isotropic microvoids model in dislocation format; the microstrain model [8]; the linear isotropic asymmetric microstretch model in dislocation format; the linear isotropic Cosserat model; the Popov-Kröner dislocation model; the symmetric earthquake structure model of Teisseyre, the gauge theory of dislocations of Lazar and Anastassiadis.

\section{Mathematical analysis}

We will rewrite the initial boundary value problem $(\mathcal{P})$ described in the previous section as an abstract Cauchy problem in a Hilbert space. Let us define the space $\mathcal{X}=\left\{w=(u, v, P, K) \mid u \in H_{0}^{1}(\Omega), \quad v \in L^{2}(\Omega), \quad P \in H_{0}(\mathrm{Curl} ; \Omega), \quad K \in\right.$ $\left.L^{2}(\Omega)\right\}$. Further, we introduce the operators $A_{1} w=v, A_{2} w=\operatorname{Div}[\mathbb{C} \cdot \operatorname{sym}(\nabla u-P)], A_{3} w=K, A_{4} w=-\operatorname{Curl}\left[\mathbb{L}_{c} \cdot \operatorname{Curl} P\right]+$ $\mathbb{C}$. $\operatorname{sym}(\nabla u-P)-\mathbb{H}$. sym $P$, where all the derivatives of the functions are understood in the sense of distributions. Let $\mathcal{A}$ be the operator $\mathcal{A}=\left(A_{1}, A_{2}, A_{3}, A_{4}\right)$ with domain $\mathcal{D}(\mathcal{A})=\{w=(u, v, P, K) \in \mathcal{X} \mid \mathcal{A} w \in \mathcal{X}\}$. With the above definitions, the problem $(\mathcal{P})$ can be transformed into the following abstract Cauchy problem in the Hilbert space $\mathcal{X}$ :

$$
\frac{d w}{d t}(t)=\mathcal{A} w(t)+\mathcal{F}(t), \quad w(0)=w_{0}, \quad \text { where } \quad \mathcal{F}(t)=(0, f, 0, M) \quad \text { and } \quad w_{0}=\left(u_{0}, \dot{u}_{0}, P_{0}, \dot{P}_{0}\right) .
$$

On $\mathrm{H}(\mathrm{Curl} ; \Omega)$ we consider the seminorm $\|P\|\left\|^{2}:=\right\| \operatorname{sym} P\left\|_{L^{2}(\Omega)}^{2}+\right\| \operatorname{Curl} P \|_{L^{2}(\Omega)}^{2}$. We have the following result:

Theorem $2.1[10-12]$ On $\mathrm{H}_{0}(\mathrm{Curl} ; \Omega)$ the norms $\|\cdot\|_{\mathrm{H}(\mathrm{Curl} ; \Omega)}$ and $\||\cdot|||$ are equivalent. In particular, ||$|\cdot|||$ is a norm on $\mathrm{H}_{0}(\mathrm{Curl} ; \Omega)$ and there exists a positive constant $c$, such that $c\|P\|_{\mathrm{H}(\mathrm{Curl} ; \Omega)} \leq\|\| P\|\|$, for all $P \in \mathrm{H}_{0}(\mathrm{Curl} ; \Omega)$.

The new coercive inequalities recently proved by Neff, Pauly and Witsch [10-12] and by Bauer, Neff, Pauly and Starke [1,2] represent the main point in establishing the desired well-posedness results:

Theorem 2.2 (Existence, uniqueness and continuous dependence result) Assume that $f, M \in C^{1}\left(\left[0, t_{1}\right) ; L^{2}(\Omega)\right)$, wo $\in$ $\mathcal{D}(\mathcal{A})$ and the fourth order elasticity tensors $\mathbb{C}, \mathbb{L}_{c}$ and $\mathbb{H}$ are symmetric and positive definite. Then, there exists a unique solution $w \in C^{1}\left(\left(0, t_{1}\right) ; \mathcal{X}\right) \cap C^{0}\left(\left[0, t_{1}\right) ; \mathcal{D}(\mathcal{A})\right)$ of the Cauchy problem $(\mathcal{P C})$. Moreover, we have the following estimate $\|w(t)\|_{\mathcal{X}} \leq\left\|w_{0}(t)\right\|_{\mathcal{X}}+C \int_{0}^{t}\left(\|f(s)\|_{L^{2}(\Omega)}+\|M(s)\|_{L^{2}(\Omega)}\right) d s$, where $C$ is a positive constant.

The micro-distortion tensor field $P \in \mathrm{H}_{0}(\mathrm{Curl} ; \Omega) \supsetneq \mathrm{H}_{0}^{1}(\Omega)$ is not necessarily smooth, it may jump across hyperplanes.

\section{References}

[1] S. Bauer, P. Neff, D. Pauly, and G. Starke. Dev-Div and DevSym-DevCurl inequalities for incompatible square tensor fields with mixed boundary conditions. submitted, 2013.

[2] S. Bauer, P. Neff, D. Pauly, and G. Starke. New Poincaré-type inequalities. C. R. Math., 352(2):163-166, 2014.

[3] W.D. Claus and A.C. Eringen. Dislocation dispersion of elastic waves. Int. J. Engng. Sci., 9:605-610, 1971.

[4] I.D. Ghiba, P. Neff, A. Madeo, L. Placidi, and G. Rosi. The relaxed linear micromorphic continuum: Existence, uniqueness and continuous dependence in dynamics. Math. Mech. Solids, doi: 10.1177/1081286513516972, 2014.

[5] A. Madeo, P. Neff, I.D. Ghiba, L. Placidi, and G. Rosi. Wave propagation in relaxed linear micromorphic continua: modelling metamaterials with frequency band-gaps. Cont. Mech. Therm., doi 10.1007/s00161-013-0329-2, 2014.

[6] P. Neff. The Cosserat couple modulus for continuous solids is zero viz the linearized Cauchy-stress tensor is symmetric. Z. Angew. Math. Mech., 86:892-912, 2006.

[7] P. Neff. Existence of minimizers for a finite-strain micromorphic elastic solid. Proc. Roy. Soc. Edinb. A, 136:997-1012, 2006.

[8] P. Neff and S. Forest. A geometrically exact micromorphic model for elastic metallic foams accounting for affine microstructure. Modelling, existence of minimizers, identification of moduli and computational results. J. Elasticity, 87:239-276, 2007.

[9] P. Neff, I.D. Ghiba, A. Madeo, L. Placidi, and G. Rosi. A unifying perspective: the relaxed linear micromorphic continua. Cont. Mech. Therm., doi 10.1007/s00161-013-0322-9, 2014.

[10] P. Neff, D. Pauly, and K.J. Witsch. Poincaré meets Korn via Maxwell: Extending Korn's first inequality to incompatible tensor fields. arXiv:1203.2744, submitted.

[11] P. Neff, D. Pauly, and K.J. Witsch. A canonical extension of Korn's first inequality to H(Curl) motivated by gradient plasticity with plastic spin. C. R. Acad. Sci. Paris, Ser. I, 349:1251-1254, 2011.

[12] P. Neff, D. Pauly, and K.J. Witsch. Maxwell meets Korn: a new coercive inequality for tensor fields in $\mathbb{R}^{N \times N}$ with square-integrable exterior derivative. Math. Methods Appl. Sci., 35:65-71, 2012.

[13] R. Picard, S. Trostorff, and M. Waurick. On some models for elastic solids with micro-structure. to appear in Z. Angew. Math. Mech., 2014. 